\title{
Blood pressure measurements within the JNC7 pre-hypertensive range after 32 weeks of gestation are a risk factor for decreased fetal growth
}

\author{
Hypertension Research (2012) 35, 128-130; doi:10.1038/hr.2011.158; published online 15 September 2011
}

Pre-hypertension (pre-HT) is defined by either a systolic blood pressure (SBP) between 130 and $139 \mathrm{~mm} \mathrm{Hg}$ or a diastolic blood pressure (DBP) between 80 and $89 \mathrm{~mm} \mathrm{Hg}$ by the seventh report of the Joint National Committee on Prevention (JNC7). The JNC7 definition of pre-HT does not, however, address pregnant women. ${ }^{1}$

Fetal growth restriction (FGR) is an important clinical problem that is known to lead to adverse perinatal outcomes including stillbirth and unexplained fetal death. Although pregnancy-induced hypertension (PIH) is associated with FGR, FGR also occurs in $\sim 8-14 \%$ of women without hypertensive disorders. $^{2,3}$ Little is known regarding whether pre-HT range pressures impact pregnancy outcomes in the absence of PIH. ${ }^{4}$ In this context, blood pressure (BP) in the preHT range may be a risk factor for FGR. To clarify this hypothesis, we investigated the relationship between a pre-HT-range BP after 32 weeks of gestation (GW) and fetal growth.

The patients in the study had referral and follow-up visits to the Kyushu University Hospital, between January 2008 and March 2010. The gestational age was confirmed by sonographic fetal measurements during the first trimester. This study was approved by the Institutional Review Board. The subjects were Japanese women and were recruited before $20 \mathrm{GW}$ and all provided informed consent. Only women who delivered after $34 \mathrm{GW}$ were included. Patients were also excluded if they had a diagnosis of any hypertension or pre-eclampsia during pregnancy, gestational or pre-existing diabetes mellitus, thyroid disorders, active collagen disease, liver or renal dysfunction, placenta previa, uterine malformations or fetal serious malformations and chromosomal aberrations. Women who smoked or who regularly consumed alcohol during pregnancy were also excluded. Finally, 474 women were enrolled. Measurements of BP and the diagnosis of pre-eclampsia were performed were based on the criteria of the American College of Obstetricians and Gynecologists. ${ }^{5}$ The subjects were classified into one of two groups, control (SBP $<130$ and DBP $<80 \mathrm{~mm} \mathrm{Hg}$ $(n=403)$ and Pre-HT (SBP 130-139 and/or DBP $80-89)(n=71)$ at after $32 \mathrm{GW}$ based on JNC7 pre-hypertensive definition for nonpregnant adults. ${ }^{1}$ The deviation from the standard birth weight was calculated using the formula derived from normal values for the Japanese population. ${ }^{6}$ Statistical analyses were performed using the unpaired $t$-test, Mann-Whitney test, and $\chi^{2}$-test, a multivariate analysis. $P$-values $<0.05$ were considered significant.

We performed a retrospective chart review on recorded maternal demographic characteristics (Table 1). There were statistically significant differences in the ratio of neonatal birth weight and the deviation from standard birth weight and the percentage of small for gestational age (SGA) for the control and pre-HT groups. There were statistically significant differences between the control and Pre-HT groups in terms of the early pregnancy SBP and DBP, and the maternal serum UA level. Then we performed a multivariate analysis for predictors of SGA $(<-2$ s.d.), which included the maternal BP and body mass index (BMI) at early and late pregnancy (Table 2). Of these, BMI at early pregnancy and DBP after 32 weeks were significant predictors of SGA. In contrast, BMI at late pregnancy was a negative predictor of SGA.
In this study we investigated the impact of maternal BP in the pre-HT range after $32 \mathrm{GW}$ on perinatal outcome, and produced the following notable findings. Women in the pre-HT group had significantly higher mean SBP and DBP readings in early pregnancy compared with the control group. Additionally, their serum UA levels were higher. DBP after 32 weeks and maternal BMI early in pregnancy were both predictors of SGA in a multivariate analysis. Pregnancy outcomes, however, between the pre-HT group and the control group were similar.

In all, two studies have alluded to a possible relationship between FGR and elevated blood pressure still within clinically normotensive levels during late pregnancy. ${ }^{7,8}$ Churchill et al. ${ }^{7}$ found that $5 \mathrm{~mm} \mathrm{Hg}$ increases in mean $24 \mathrm{~h} \mathrm{DBP}$ at 28 and $36 \mathrm{GW}$ were associated with 68 and $76 \mathrm{~g}$ decreases in birth weight, respectively. The associations between ambulatory SBP and indices of fetal growth, however, were weak and inconsistent. The ambulatory blood pressure at $18 \mathrm{GW}$ did not predict fetal growth. The study also showed a continuous inverse association between fetal growth and maternal BP, over the levels seen in a normal pregnancy. ${ }^{8}$ Tranquilli et al. reported that women with idiopathic FGR had higher mean DBP and SBP levels on 24-h ambulatory BP monitoring than controls, although these levels were still in the normotensive range. ${ }^{9}$ In all these earlier studies, BP was measured by 24-h ambulatory recordings, whereas we measured BP at a routine clinic visit. Despite this difference, our results were very similar to the previous reports. As it is difficult to incorporate 24-h ambulatory $\mathrm{BP}$ recordings into the normal clinical practice, the finding of an increase in DBP at a routine visit should prompt additional blood pressure monitoring 
Table 1 Clinical profiles and outcomes of subjects

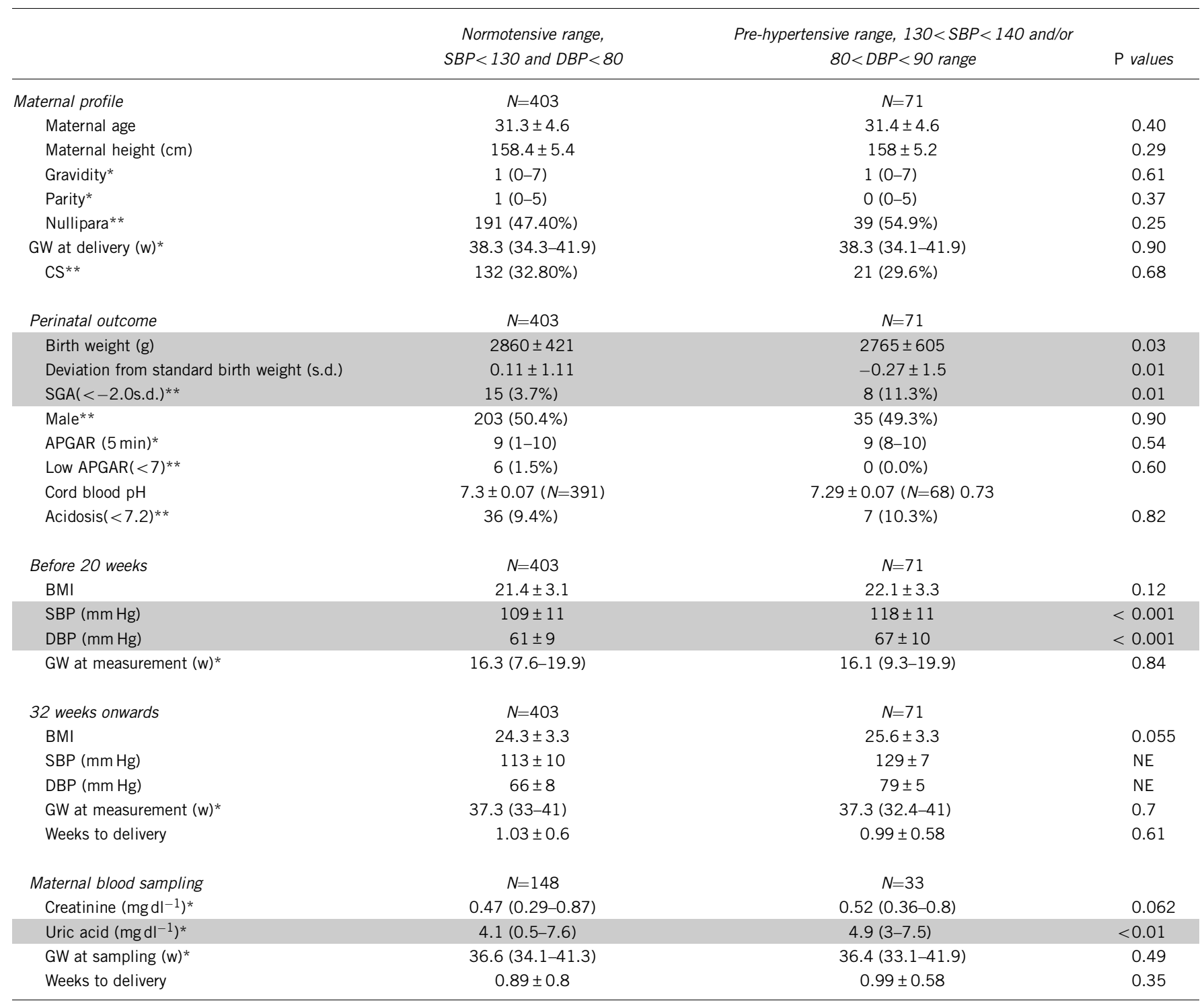

Abbreviations: BMI, body mass index; DBP, diastolic blood pressure; GW, weeks of gestation; SBP, systolic blood pressure; SGA, small for gestational age.

Data are expressed as either the mean \pm s.d., * median (range) or ** number of cases (\%). The $P$-values for the comparison between normotensive vs. pre-HT levels were calculated using either the unpaired $t$-test, *Mann-Whitney test, ** $\chi^{2}$-test (GraphPad Prism 5, GraphPad Software, CA, USA). $P$-values $<0.05$ were considered significant. Gray shaded values showed statistically significance.

Table 2 Multivariate analysis for SGA( $<-2$ s.d. $)$

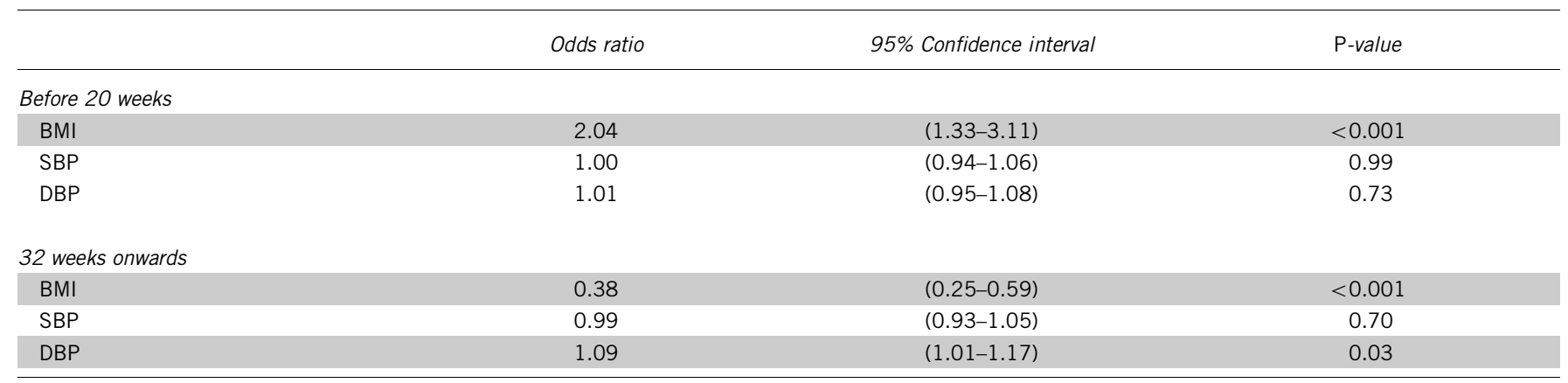

Abbreviations: BMI, body mass index; DBP, diastolic blood pressure; SBP, systolic blood pressure; SGA, small for gestational age.

Multivariate analysis of six predictors of SGA (deviation from mean birth weight $<-2.0$ ) was performed using EXCEL Tokei 2010 -in Japanese (Shakai Joho Service, Tokyo, Japan). P-values $<0.05$ were considered significant. Gray shaded values showed statistically significance. 
and if persistent, further assessment of fetal growth.

BMI was a significant predictor of SGA in the multivariate analysis. Interestingly, an elevated BMI in early pregnancy was a risk factor for decreased fetal growth, whereas an increased BMI in late pregnancy protected against SGA. The reason for this effect remains unclear as maternal BMI and fetal body weight have an established positive correlation. ${ }^{9}$ These findings may indicate that an increased BMI in early pregnancy may be a risk factor for later DBP elevation, or alternatively, other interacting factors may be involved. The significance of an isolated UA elevation is unclear. Several observations suggest that serum UA level is a measurable indicator of underlying oxidative stress. ${ }^{10}$ In this study, maternal serum UA levels were increased significantly in the preHT patients. Taken together, these findings suggest that oxidative stress may be present even in the absence of PIH.

A larger prospective study is necessary to confirm the relationship between pre-HTrange blood pressures, fetal growth and adverse perinatal outcomes, because this study is a retrospective and observational study from a single institution with the subjects all being Japanese women.

\section{CONFLICT OF INTEREST}

The authors declare no conflict of interest.

\section{Acknowledgements}

This work was supported in part by a grantin-aid from the Japan Ministry of Education (23561596).

Kotaro Fukushima $^{1}$, Seiichi Morokuma ${ }^{1}$, Kiyomi Tsukimori $^{2}$, Masaharu Murata ${ }^{1}$ and Norio Wake ${ }^{1}$

${ }^{1}$ Department of Obstetrics and Gynecology, Kyushu University Hospital, Kyushu University, Fukuoka, Japan and

${ }^{2}$ Department of Obstetrics, Fukuoka Children's Hospital, Fukuoka, Japan E-mail:kfuku@gynob.med.kyushu-u.ac.jp

1 The Seventh Report of the Joint National Committee on Prevention, Detection, Evaluation, and Treatment of High Blood Pressure [JNC 7 Express] U.S. Department of Health and Human Services National Institutes of Health, National Heart, Lung, and Blood Institute,
National High Blood Pressure Education Program, NIH Publication No. 04-5230.

2 James JL, Whitley GS, Cartwright JE. Pre-eclampsia: fitting together the placental, immune and cardiovascular pieces. J Pathol 2010; 221: 363-378.

3 Mandruzzato G, Antsaklis A, Botet F, Chervenak FA, Figueras F, Grunebaum A, Puerto B, Skupski D, Stanojevic M. Intrauterine restriction (IUGR). J Perinat Med 2008; 36: 277-281.

4 Villar J, Carroli G, Wojdyla D, Abalos E, Giordano D, Ba'aqeel $\mathrm{H}$, Farnot $\mathrm{U}$, Bergsjo $\mathrm{P}$, Bakketeig L, Lumbiganon $\mathrm{P}$, Campodónico L, Al-Mazrou Y, Lindheimer M, Kramer M. Preeclampsia, gestational hypertension and intrauterine growth restriction, related or independent conditions? Am J Obstet Gynecol 2006; 194: 921-931.

5 American College of Obstetricians and Gynecologists. Hypertension in pregnancy. ACOG Technical Bulletin No. 219 The College: Washington, DC, 1996.

6 Shinozuka N. Fetal biometry and fetal weight estimation: JSUM standardization. Ultrasound Rev Obset Gynecol 2002; 2: 156-161.

7 Churchill D, Perry IJ, Beevers DG. Ambulatory blood pressure in pregnancy and fetal growth. Lancet 1997; 349: 7-10.

8 Tranquilli AL, Giannubilo SR. Blood pressure is elevated in normotensive pregnant women with intrauterine growth restriction. Eur J Obstet Gynecol Reprod Biol 2005; 122: 45-48.

9 Xue F, Willett WC, Rosner BA, Forman MR, Michels KB. Parental characteristics as predictors of birthweight. Hum Reprod 2008; 23: 168-177.

10 Fukushima K, Murata M, Tsukimori K, Eisuke K, Wake N. 8-Hydroxy-2-Deoxyguanosine Staining in Placenta Is Associated With Maternal Serum Uric Acid Levels and Gestational Age at Diagnosis in Pre-Eclampsia. Am J Hypertens 2011; 24: 829-834. 